\title{
Proton energy spectra during ground level enhancements as measured by EPHIN aboard SOHO
}

B. Heber*

Christian-Albrechts-University Kiel, Germany

E-mail: heber@physik.uni-kiel.de

\section{N.Dresing}

Christian-Albrechts-University Kiel, Germany

\section{K. Herbst}

Christian-Albrechts-University Kiel, Germany

\section{A. Klassen}

Christian-Albrechts-University Kiel, Germany

\section{P. Kühl}

Christian-Albrechts-University Kiel, Germany

\section{R. Gomez-Herrero}

\section{Universidad de Alcala}

Ground Level Enhancements (GLEs) are solar energetic particle (SEP) events that are recorded by ground-based instrumentation. The energy of the particles is so high that they produce secondary particles in the Earth's atmosphere, i.e. protons and neutrons, which are detected as sudden increases in cosmic ray intensities measured by e.g. neutron monitors. Since the launch of SOHO in December 1995 the neutron monitor network recorded 16 GLEs. The Electron Proton Helium INstrument on board SOHO has been designed to measure protons and helium up to 53 $\mathrm{MeV} /$ nucleon as well as electrons up to $8.3 \mathrm{MeV}$. Above these energies, particles penetrate all detector elements and thus, a separation between different particle species becomes more complicated. Recently we developed a method that allows deriving the energy spectrum for penetrating protons up to more than $1 \mathrm{GeV}[1,2]$. In this contribution we present the proton energy spectra for two of the 16 above-mentioned GLEs and compare them to previous measurements (.e.g. [7, 17]). Although there are differences of up to a factor two the overall shape of the energy spectra agree surprisingly well. Thus it has been demonstrated that EPHIN measurements are a valuable tool for understanding GLE.

The 34th International Cosmic Ray Conference,

30 July- 6 August, 2015

The Hague, The Netherlands

${ }^{*}$ Speaker. 


\section{Introduction}

Forbush [3] reported the first Solar Energetic Particle (SEP) event that is now called a groundlevel enhancement (GLE). They are produced when ions with energies above several hundred of $\mathrm{MeVs}$ create a nuclear cascade in the Earth's atmosphere. As a consequence detectors at ground level measure an increase above the galactic cosmic ray background. Since than 71 GLEs have been reported. The largest measured increase of about $4500 \%$ was observed during GLE 5 on February 23, 1956 [4]. During solar cycle 23 and 2416 event was measured by more than one neutron monitor. Aside understanding the physics of GLEs, i.e. the acceleration process, the recent paper by [5] claim among other the January 6, 2014 event to be a GLE. The dilemma of the definition whether a solar energetic particle event is recorded as a GLE or not depends on several factors i.e. the measurement capabilities. Measurements below the atmospheric shielding i.e. at positions with magnetic cutoff rigidities less than a GV have thus to rely on spacecraft measurements outside the Earth atmosphere i.e. the Payload for Antimatter Matter Exploration and Light-Nuclei Astrophysics (PAMELA). However, observations made inside the magnetosphere suffer from the varying cutoff rigidity along its trajectory (see [6]). In contrast observations made in interplanetary space are mainly restricted in the energy range as well as in statistical accuracy Here we utilize published data from the PAMELA experiment that has recorded proton spectra during different phases of the December 13, 2006 event [7] and compare the spectra with the ones derived form the Kiel Electron Proton Helium Instrument (EPHIN) aboard the SOHO spacecraft [1].

\section{Instrumentation}

The Electron Proton Helium INstrument (EPHIN), as displayed in Fig. 1, consists of a stack of silicon solid state detectors (SSDs, A-F) surrounded by an anticoincidence (scintilator, G). Particles with energies above $\sim 100 \mathrm{MeV} /$ Nucleon and above $\sim 300 \mathrm{MeV} /$ Nucleon penetrate the entire instrument from the front and from the back, respectively. Unfortunately EPHIN provides no information of the directionality of the measured particles. Only in a small energy window one can distinguish between particles entering the instrument from the front ("forward particles", entering at detector A, exiting at detector F) and particles entering the instrument from behind ("backward particles", entering at detector F, exiting at detector A). Since backward particles first have to penetrate the entire spacecraft, they will reach the actual instrument with only a fraction of their initial energy which leads to systematic uncertancies in the derived spectra (c.f. [1] for details). To take this effects into account, a GEANT4 simulation has been set up, including a $10 \mathrm{~cm}$ aluminium block behind the instrument to mimic the spacecraft shielding[1]. To calculate the initial total energy of a penetrating particle based on the energy deposition in the detector, mono-energetic simulations with isotropic fluxes of protons, electrons and helium particles in front of (for forward particles) and behind the instrument (backward particles) have been performed individually. Results for protons and helium particles are shown in fig. 1, right. The figure shows the resulting counts (color-coded) as a function of the energy-loss and the initial total energy. For the energy loss, the minimum of the energy deposition in either the $\mathrm{C}$ or $\mathrm{D}$ detector is used to reduce noise based on the statistical nature of the energy loss (c.f. [1]). For both, protons and helium particles, the forward particles form a 

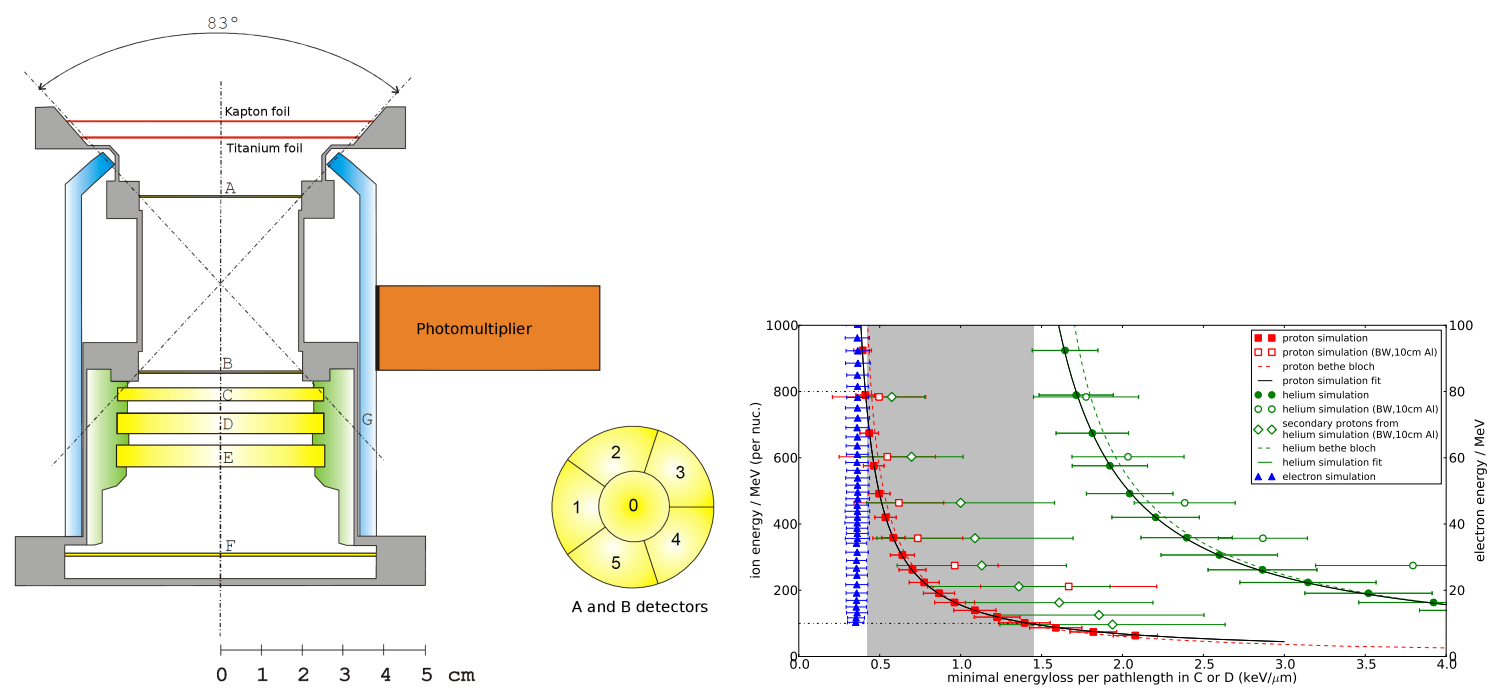

Figure 1: The left panel shows a sketch of the EPHIN instrument [8]. The right panel displays the relation between initial total energy and the minimum of the energy loss in detector $\mathrm{C}$ and $\mathrm{D}$ for protons (red), helium particles (green) and electrons (blue, right hand axis). The maximum energy of electrons is chosen to be $100 \mathrm{MeV}$ [1] Open symbols represent results from backward simulations, secondary protons caused by helium (green diamonds) are shown individually.

narrow population with a clear relation between total energy and energy loss, as expected based on the Bethe-Bloch equation. The backward particles on the other hand can cause a wide variety of energy losses due to the interaction in the spacecraft. Furthermore, at low energies the energy loss is enhanced as backward particles at this energies loose a significant fraction of their energy in the shielding. In addition, the distribution of backward helium particles shows a secondary population at typical energy losses of protons, which are caused by secondary protons created in the shielding via nuclear interactions. As discussed in [1] the energy loss regions can be divided in three regimes: The first one is the given by energy losses less than $\sim 0.4 \mathrm{MeV}$ that is dominated by relativistic protons and electrons. The second regime is determined by energy losses in the range of $0.4 \mathrm{MeV}$ to $1.4 \mathrm{MeV}$. This range has been shown to be useful in order to investigate the proton spectra in the range of above $100 \mathrm{MeV}$ to more than $1 \mathrm{GeV}$ (cf. [1, 2]). Note, that relativistic Helium is a major contribution for energy losses above 1.2 MeV. However, it was shown by Kühl et al. [1] that for solar energetic particle events with a helium to proton ratio of $10 \%$ this effect can be neglected due to the shape of the energy spectra. In this contribution we analyze the variation of the energy spectra during the two ground level enhancements 70 and 69. For the first, the December 13, 2006 event (left panel of Fig. 2), the PAMELA investigation has published the energy spectra during different phases of the event [7].

\section{Analysis}

The GLE 70 on December 13, 2006 has been studied extensively by different authors (c.f. $[9,10,11,12,7,13]$. An unusual large near-solar-minimum GLE was observed after a 4B solar flare at 02:20 UT, at S06 W24, associated with an X3.4 X-ray event and type II and IV radio bursts. 

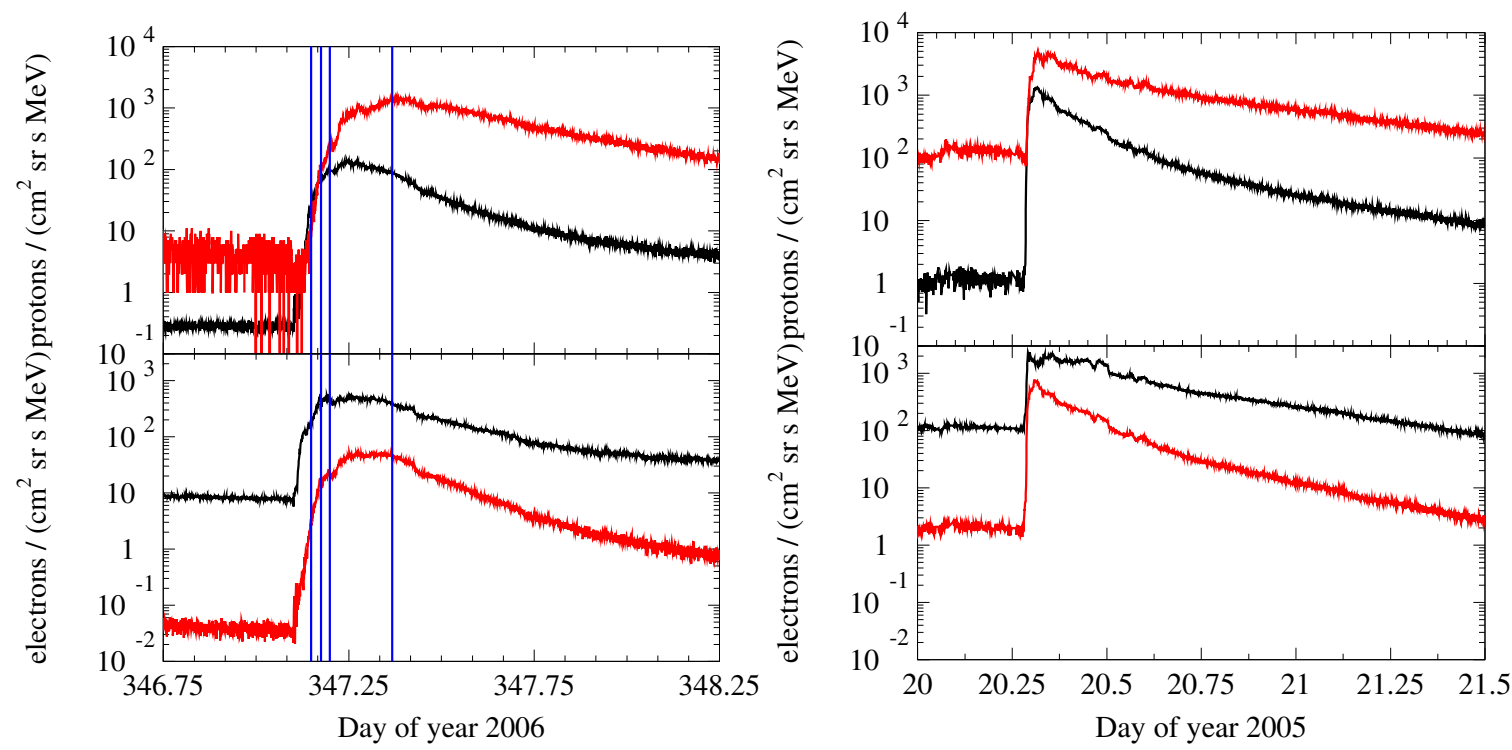

Figure 2: Upper and lower panels shows 1 minute averaged count rate profiles of the integral channel and $\sim 30 \mathrm{MeV}$ protons as well as $\sim 1$ and $\sim 4 \mathrm{MeV}$ electrons observed during December 13, 2006 (left) and January 20, 2005 (right), respectively. Marked by the blue lines are time periods for which energy spectra measured by PAMELA are published [7].

A fast full-halo CME with a velocity of $1500 \mathrm{~km} / \mathrm{s}$ ) had been reported by the LASCO observations (http://sgd.ngdc.noaa.gov/sgd/jsp/solarindex.jsp). The 1 minute averaged time profile of the integral channel and $\sim 30 \mathrm{MeV}$ protons as well as $\sim 1$ and $\sim 4 \mathrm{MeV}$ electrons observed during December 13, 2006 is displayed in the left panel of Fig. 2. Proton spectra by the PAMELA instrument have been measured during different times of the event. From these periods we compared the PAMELA results with the ones from our instrument for the period:

1. from $3: 18$ to $3: 49 \mathrm{UT}$,

2. from 3:49 to $4: 33 \mathrm{UT}$,

3. from $4: 33$ to $4: 59 \mathrm{UT}$ and

4. from 8:18 to 9:17 UT.

Fig. 3 displays the energy spectra from PAMELA (green dots) together with the SOHO EPHIN and if available with the GOES/neutron monitor measurements. The red dots and black dots are intensities determined by assuming that all particles are entering the telescope from the front or that from a certain threshold onward particles from the back can contribute. These values can be seen as the upper and lower limits for the measured intensities. All energy spectra are corrected for background by subtracting the energy spectra obtained in November 2006. The first two, the third and forth spectra were obtained during the rising phase, during the maximum and the declining phase of the event, respectively (see Fig.2). While Fig. 3 shows a good agreement of the SOHO data with the ones obtained by PAMELA and by [11] during the first two periods, the difference are becoming larger during the maximum and the declining phase of the event. The reason for such 

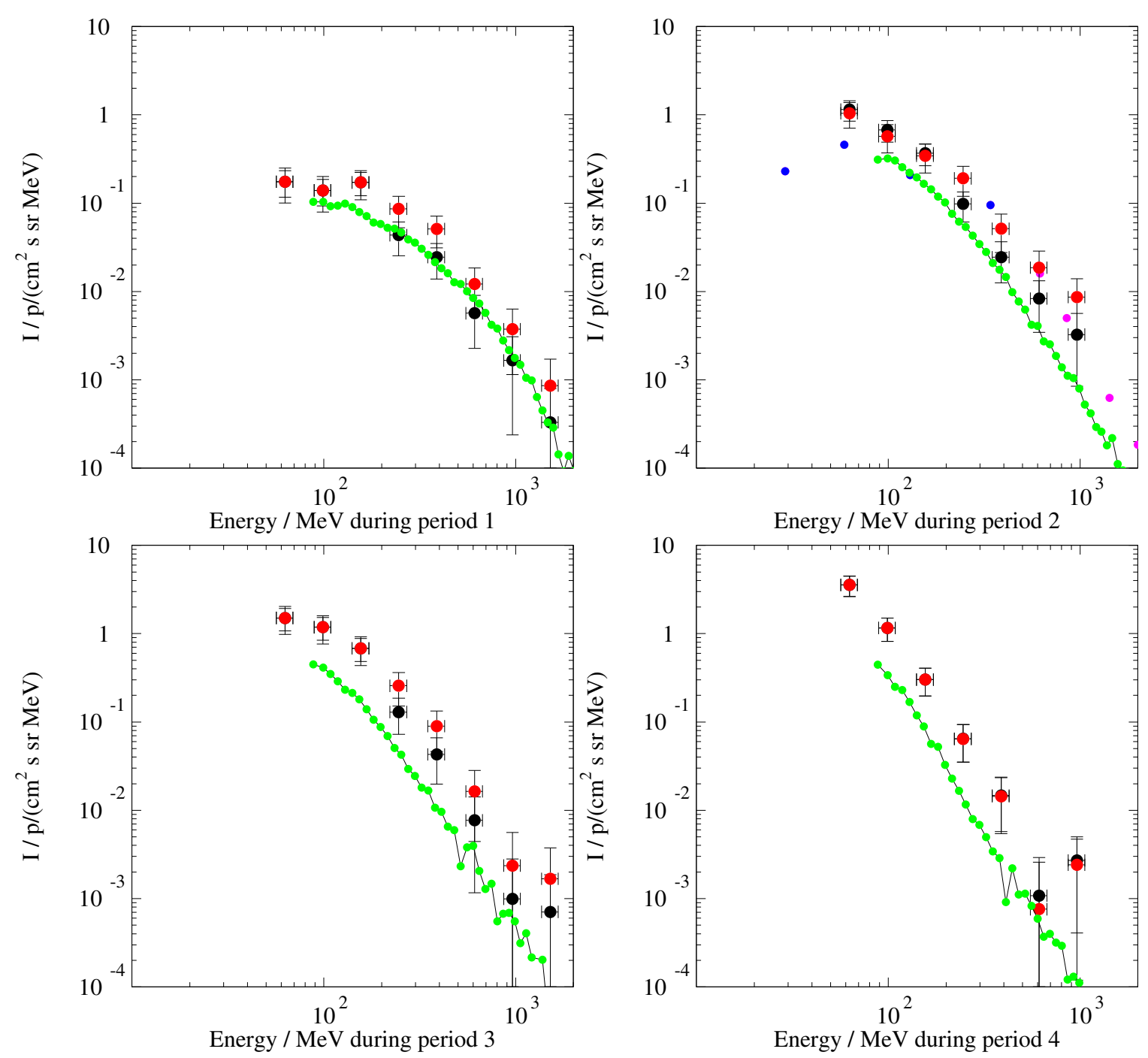

Figure 3: Proton energy spectra during four time periods on December 13 and 14, 2006. The panel compare the EPHIN measurements (black and red dots) with the PAMELA observations (green dots) from Adriani et al. [7]. The blue and magenta dots have been extracted from [11]. The blue and magenta dots in the upper right panel are derived from GOES and neutron monitor measurements, respectively.

a behavior could have different reasons that need to will be investigated in the future. However, during the second period we compared the measurements in addition to neutron monitor and GOES observations. Although all results are in good agreement with each other we find a tendency that the neutron monitor data are in better agreement with our measurements than with the published PAMELA results.

In order to investigate the measurement capabilities of the EPHIN experiment further we analyzed the energy spectra during the January 20, 2005 GLE. This GLE has been described in the literature by several authors $[14,13,15,16,17]$. The intensity time profiles of protons and electron during GLE 69 are displayed in the right panel of Fig. 2. The energy spectra obtained for the event are determined close to the intensity maximum at 6:55 UT and during the declining phase of the 

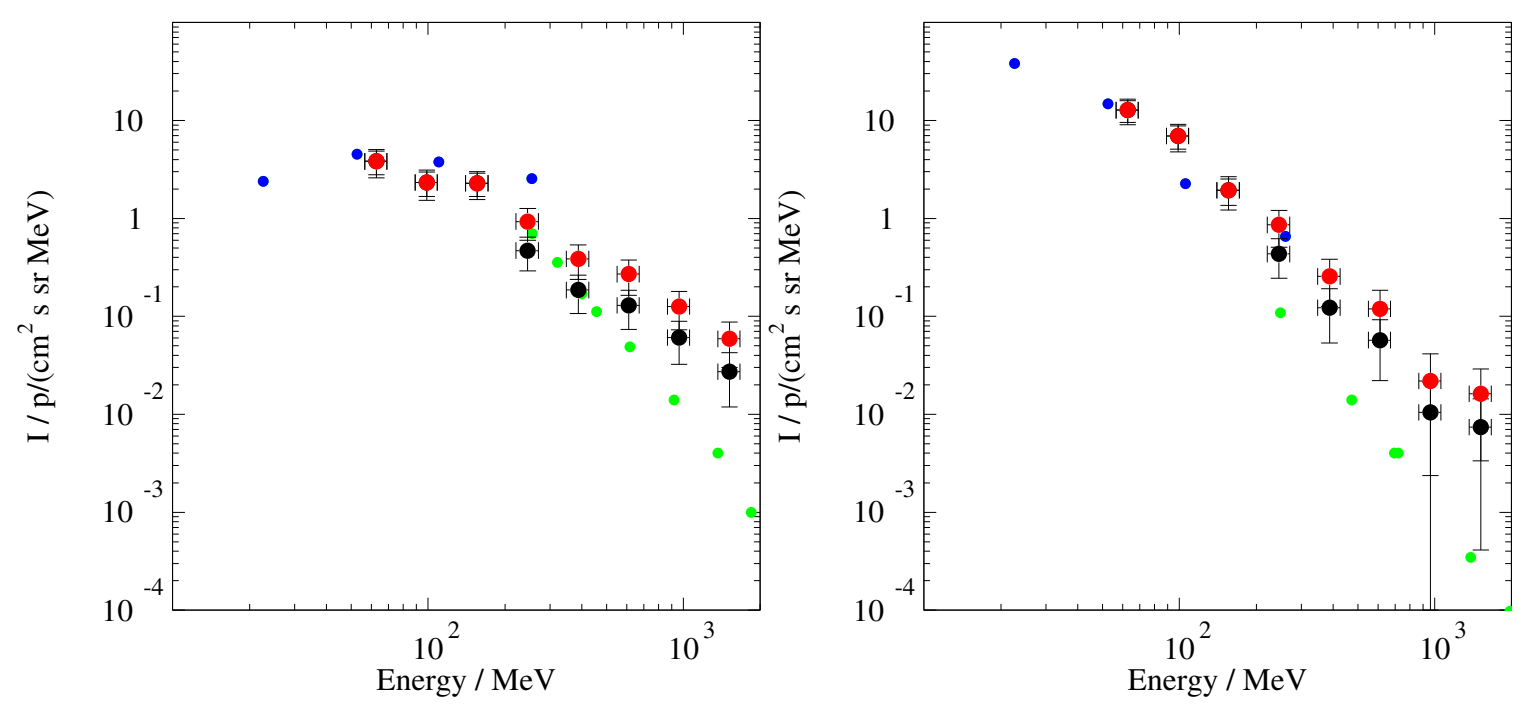

Figure 4: Proton energy spectra during the maximum time of the January 20, 2005 GLE neutron monitor at 6:55 UT (left) and during the declining phase of the event at 9:00 UT (right). Due to statistical limitation EPHIN data are averaged from 6:50 to 7:10 UT and 8:45 to 9:15 UT The panel compare the EPHIN measurements (black and red dots) with the one from neutron monitor and GOES measurements. The green dots have been extracted from [17].

GLE event at 9:00 UT. The green dots have been extracted from [17]. As for the GLE 70 we find a better agreement during the onset of the event than during the maximum phase. But in contrast to the other event a deviation of the shape of the energy spectra at energies above $700 \mathrm{MeV}$ is found. The SOHO spectra are much flatter than the ones obtained from the neutron monitors. The reason for this behavior becomes obvious from Fig. 1. High energy protons are associated with low energy losses. Although the major contribution from electrons should be below $0.4 \mathrm{MeV}$ there exist a tail in the electron distribution ranging to values above $0.4 \mathrm{MeV}$. Thus there is a potential cross talk that only depends on the electron spectrum. Thus further investigations are needed.

\section{Summary and Conclusion}

We have determined SOHO/EPHIN energy spectra of protons in the energy range from below $100 \mathrm{MeV}$ to above $1 \mathrm{GeV}$ for GLE 69 and 70. By comparing the data with PAMELA, neutron monitor network and GOES data we could successfully show here that these extended measurement capabilities of SOHO/EPHIN is a valuable tool for investigating energetic particle events that are associated with increased proton fluxes at energies of above $100 \mathrm{MeV}$, as provided by e.g. SEPServer (www.sepserver.eu).

\section{Acknowledgments}

We are grateful to the PAMELA team for making their data publicly available. The SOHO/EPHIN project is supported under Grant 50 OC 1302 by the German Bundesministerium für Wirtschaft through the Deutsches Zentrum für Luft- und Raumfahrt (DLR). P.K. has received funding from 
the European Union's Horizon 2020 research and innovation programme under grant agreement No 637324.

\section{References}

[1] P. Kühl, S. Banjac, N. Dresing, R. Goméz-Herrero, B. Heber, A. Klassen, and C. Terasa, Proton intensity spectra during the solar energetic particle events of May 17, 2012 and January 6, 2014, Astronomy and Astrophysics 576 (Apr., 2015) A120.

[2] P. Kühl, N. Dresing, J. Gieseler, B. Heber, and A. Klassen, Galactic cosmic ray quiet time spectra from $300 \mathrm{MeV}$ up to above $1 \mathrm{GeV}$ measured with SOHO/EPHIN, 2015.

[3] S. E. Forbush, Three Unusual Cosmic-Ray Increases Possibly Due to Charged Particles from the Sun, Physical Review 70 (Nov., 1946) 771-772.

[4] D. V. Reames, The Two Sources of Solar Energetic Particles, Space Science Reviews 175 (June, 2013) 53-92, [arXiv:1306.3608].

[5] N. Thakur, N. Gopalswamy, H. Xie, P. Makela, S. Yashiro, S. Akiyama, and J. M. Davila, Ground Level Enhancement in the 2014 January 6 Solar Energetic Particle Event, ArXiv e-prints (June, 2014) [arXiv:1406.7172].

[6] P. Picozza, A. M. Galper, G. Castellini, O. Adriani, F. Altamura, M. Ambriola, G. C. Barbarino, A. Basili, G. A. Bazilevskaja, R. Bencardino, M. Boezio, E. A. Bogomolov, L. Bonechi, M. Bongi, L. Bongiorno, V. Bonvicini, F. Cafagna, D. Campana, P. Carlson, M. Casolino, C. de Marzo, M. P. de Pascale, G. de Rosa, D. Fedele, P. Hofverberg, S. V. Koldashov, S. Y. Krutkov, A. N. Kvashnin, J. Lund, J. Lundquist, O. Maksumov, V. Malvezzi, L. Marcelli, W. Menn, V. V. Mikhailov, M. Minori, S. Misin, E. Mocchiutti, A. Morselli, N. N. Nikonov, S. Orsi, G. Osteria, P. Papini, M. Pearce, M. Ricci, S. B. Ricciarini, M. F. Runtso, S. Russo, M. Simon, R. Sparvoli, P. Spillantini, Y. I. Stozhkov, E. Taddei, A. Vacchi, E. Vannuccini, S. A. Voronov, Y. T. Yurkin, G. Zampa, N. Zampa, and V. G. Zverev, PAMELA A payload for antimatter matter exploration and light-nuclei astrophysics, Astroparticle Physics 27 (Apr., 2007) 296-315, [astro-ph/ 0608697 ].

[7] O. Adriani, G. C. Barbarino, G. A. Bazilevskaya, R. Bellotti, M. Boezio, E. A. Bogomolov, L. Bonechi, M. Bongi, V. Bonvicini, S. Borisov, S. Bottai, A. Bruno, F. Cafagna, D. Campana, R. Carbone, P. Carlson, M. Casolino, G. Castellini, L. Consiglio, M. P. De Pascale, C. De Santis, N. De Simone, V. Di Felice, V. Formato, A. M. Galper, L. Grishantseva, W. Gillard, G. Jerse, A. V. Karelin, S. V. Koldashov, S. Y. Krutkov, A. N. Kvashnin, A. Leonov, V. Malakhov, L. Marcelli, A. G. Mayorov, W. Menn, V. V. Mikhailov, E. Mocchiutti, A. Monaco, N. Mori, N. Nikonov, G. Osteria, F. Palma, P. Papini, M. Pearce, P. Picozza, C. Pizzolotto, M. Ricci, S. B. Ricciarini, R. Sarkar, L. Rossetto, M. Simon, R. Sparvoli, P. Spillantini, Y. I. Stozhkov, A. Vacchi, E. Vannuccini, G. Vasilyev, S. A. Voronov, J. Wu, Y. T. Yurkin, G. Zampa, N. Zampa, and V. G. Zverev, Observations of the 2006 December 13 and 14 Solar Particle Events in the $80 \mathrm{MeV} n^{-1}-3 \mathrm{GeV} n^{-1}$ Range from Space with the PAMELA Detector, Astrophysical Journal 742 (Dec., 2011) 102, [arXiv:1107.4519].

[8] R. Müller-Mellin, H. Kunow, V. Fleißner, E. Pehlke, E. Rode, N. Röschmann, C. Scharmberg, H. Sierks, P. Rusznyak, S. McKenna-Lawlor, I. Elendt, J. Sequeiros, D. Meziat, S. Sanchez, J. Medina, L. Del Peral, M. Witte, R. Marsden, and J. Henrion, COSTEP - Comprehensive Suprathermal and Energetic Particle Analyser, Solar Physics 162 (Dec., 1995) 483-504.

[9] J. W. Bieber, J. Clem, P. Evenson, and et al., A Maverick GLE: The Relativistic Solar Particle Event of December 13, 2006, International Cosmic Ray Conference 1 (2008) 229-232. 
[10] D. A. Timashkov, Y. V. Balabin, V. V. Borog, and et al., Ground-Level Enhancement of December 13, 2006 in muon hodoscopes data, International Cosmic Ray Conference 1 (2008) 209-212.

[11] E. V. Vashenyuk, G. A. Bazilevskaya, Y. V. Balabin, and et al., The GLE of December 13, 2006 according to the ground level and balloon observations, International Cosmic Ray Conference $\mathbf{1}$ (2008) 221-224.

[12] C. Plainaki, H. Mavromichalaki, A. Belov, E. Eroshenko, and V. Yanke, Modeling the solar cosmic ray event of 13 December 2006 using ground level neutron monitor data, Advances in Space Research 43 (Feb., 2009) 474-479.

[13] H. Moraal and K. G. McCracken, The Time Structure of Ground Level Enhancements in Solar Cycle 23, Space Science Reviews 171 (Oct., 2012) 85-95.

[14] D. Matthiä, B. Heber, G. Reitz, M. Meier, L. Sihver, T. Berger, and K. Herbst, Temporal and spatial evolution of the solar energetic particle event on 20 January 2005 and resulting radiation doses in aviation, Journal of Geophysical Research 114 (Aug., 2009) 8104.

[15] R. Bütikofer, E. O. Flückiger, L. Desorgher, M. R. Moser, and B. Pirard, The solar cosmic ray ground-level enhancements on 20 January 2005 and 13 December 2006, Advances in Space Research 43 (Feb., 2009) 499-503.

[16] K. G. McCracken, H. Moraal, and P. H. Stoker, Investigation of the multiple-component structure of the 20 January 2005 cosmic ray ground level enhancement, Journal of Geophysical Research (Space Physics) 113 (Dec., 2008) 12101.

[17] J. W. Bieber, J. Clem, P. Evenson, R. Pyle, A. Sáiz, and D. Ruffolo, Giant Ground Level Enhancement of Relativistic Solar Protons on 2005 January 20. I. Spaceship Earth Observations, Astrophysical Journal 771 (July, 2013) 92. 